\title{
Numerical Analysis of Laminar Natural Convection in a Quadrantal Cavity with a Solid Adiabatic Fin Attached to the Hot Vertical Wall
}

\author{
P. K. Bose, D. Sen ${ }^{\dagger}$, R. Panua and A. K. Das \\ Department of Mechanical Engineering, National Institute of Technology Agartala, India \\ $\dagger$ Corresponding Author Email: dipak_sen@ymail.com
}

(Received February 22, 2012; accepted September 20, 2012)

\begin{abstract}
Numerical analysis of laminar natural convection in a quadrantal cavity filled with water having finned hot and cold on adjacent walls have been made to examine heat and fluid flow. Numerical solutions are obtained using a commercial computational fluid dynamics package, FLUENT, using the finite volume method. Effects of the Rayleigh number, Ra, non-dimensional fin location and non-dimensional fin length on the Nusselt number, $\mathrm{Nu}$, as well as stream function and temperature fields are investigated for the range of Ra from $10^{4}$ to $10^{7}$.
\end{abstract}

Keywords: Natural convection, Quadrantal cavity, adiabatic fin, Rayleigh number, Nusselt number.

\section{NOMENCLATURE}

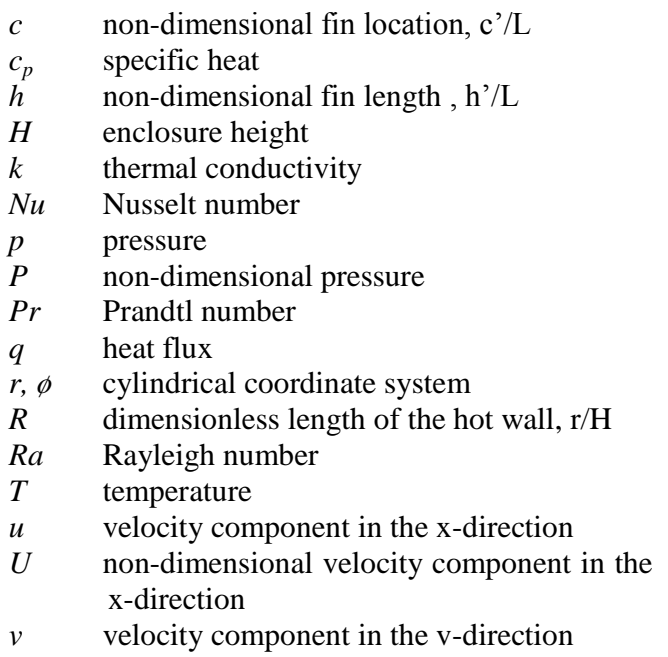

\section{INTRODUCTION}

Natural convection phenomena in enclosures filled with fluids have been an interesting research topic in recent decades since they play a vital role in many engineering applications. Application areas include solar energy collection, nuclear energy, cooling of electronic components, microelectromechanical systems, lubricating grooves, etc. Besides its importance in such processes, due to the coupling of fluid flow and energy transport, the phenomenon of natural convection remains an interesting field of investigation.
$V \quad$ non-dimensional velocity component in the y-direction

$x, y \quad$ Cartesian coordinate system

$X, Y$ non-dimensional coordinates

$\Psi \quad$ non-dimensional stream function $(=\psi / \alpha)$

$\psi \quad$ stream function

$\rho \quad$ density

$\beta \quad$ co-efficient of thermal expansion

$v \quad$ kinematic viscosity

$\alpha \quad$ thermal diffusivity

$\mu \quad$ dynamic viscosity

$\theta$ dimensionless temperature

$c \quad$ cold wall

$h \quad$ hot wall

max maximum

$\min$ minimum

The existing literature presents a vast number of studies on natural convection in enclosures. However, most of these studies have been related to either a vertically or a horizontally imposed heat flux or temperature difference. There is little work regarding natural convection in enclosures with differentially heated neighboring horizontal and vertical walls. November and Nansteel (1987) performed a numerical study on steady natural convection in a square water-filled enclosure heated from below and cooled from the adjacent wall. It is found that the first contribution to the convective heat transfer occurs at order $R a^{2}$ and convective heat transfer is shown to be most significant when slightly less than half of the lower surface is 
heated. Ganzarolli and Milanez (1995) numerically simulated steady natural convection in an enclosure heated from below and symmetrically cooled from the sides. The Rayleigh number based on the cavity height is varied from $10^{3}$ to $10^{7}$. Values of 0.7 and 7.0 for the Prandtl number are considered. The aspect ratio $L / H$ (length to height of the enclosure) is varied from 1 to 9 . Numerical values of the Nusselt number as a function of the Rayleigh number are reported, and the Prandtl number is found to have little influence on the Nusselt number. Aydin et al. (2011), studied natural convection in a quadrantal cavity heated and cooled on adjacent walls. Effects of the Rayleigh number, $\mathrm{Ra}$, on the Nusselt number, $\mathrm{Nu}$, as well as velocity and temperature fields are investigated for the range of $\mathrm{Ra}$ from $10^{3}$ to $10^{7}$. Aydin et al. (2011) also studied natural convection in an inclined quadrantal cavity heated and cooled on adjacent walls. The effects of the inclination angle, $\phi$ and the Rayleigh number, Ra on fluid flow and heat transfer are investigated for the range of angle of inclination between $0^{\circ} \leq \phi \leq 360^{\circ}$, and Ra from $10^{5}$ to $10^{7}$. It is disclosed that heat transfer changes dramatically according to the inclination angle which affects convection currents inside, i.e. flow physics inside.

Compared to extensive investigations of the natural convection in cavities with smooth walls, studies on the natural convection in cavities with partitions on the walls are limited. The characteristics of the natural convection flow significantly changed when partitions were added to the walls of rectangular cavities. For example, Shi and Khodadadi (2013) indicated that a partition attached to the heated vertical wall of a square cavity degraded the heat transfer capacity on the wall. The presence of the partition modified the boundary layer flow along the walls of the cavity. Two competing mechanisms were attributed for the flow and thermal modifications: (i) hydrodynamics blockage effect and (ii) the heat transfer characteristics from the partition. Bilgen (2002) observed that the natural convection flow circulation was blocked by partitions which were attached to the adiabatic horizontal walls of a rectangular cavity. The reduction of the heat transfer was dependent on the number and position of partitions as well as the aspect ratio of the cavity. Previous investigations have indicated that changes in the height, position and number of partitions affected the characteristics of the natural convection flow in cavities. Heretofore, most previous investigations have mainly focused on the natural convection in partitioned rectangular cavities with either adiabatic or insulated or conducting horizontal walls. Under these thermal boundary conditions, the partition directly suppressed the motion of the boundary layer along the walls or had an extra heating effect, resulting in changes in natural convection flow compared to that in cavities without partitions. There have been nearly no studies on partitioned cavities with temperature differences in both the horizontal and vertical directions, although results from cavities with smooth walls suggest that the temperature difference between the horizontal walls of a partitioned cavity would result in changes in the characteristics of natural convection in the cavity.
Oosthuizen and Paul (1985) studied differentially heated cavities with aspect ratio between 3 and 7 with a horizontal plate attached to the center of the cold vertical wall. They found the local heat transfer rate on the upper portion of the hot wall increased, but the heat transfer rate near the center of the hot wall decreased. Frederick studied a similar situation in a inclined cavity with diathermal partition at Rayleigh number between $10^{3}$ and $10^{5}$. The partition was attached to the cold wall at its center; its relative length was 0.25 and 0.50 . The inclination angle was from $90^{\circ}$ (corresponding to horizontal partition) to $45^{\circ}$. His results showed that the partition caused suppression of convection and the heat transfer relative to that in an identical cavity without partition was reduced considerably. Frederick and Valencia studied natural convection in a square cavity with a conducting partition at the center of its hot wall and with perfectly conducting horizontal walls. Partition length and its conductivity were variable. For low values of relative conductivity, they reported reduced heat transfer with respect to that in an identical cavity without partition at Rayleigh numbers between $10^{4}$ and $10^{5}$. Nag et al. (1993) studied numerically in a differentially heated square cavity where a horizontal plate was attached on the hot wall. The length and the position of the partition were varied and Rayleigh was between $10^{3}$ and $10^{6}$. They considered two cases, one with adiabatic partition and the other with perfectly conducting partition. They found that with the perfectly conducting partition the heat transfer at the cold wall increased irrespective of its position or length and it is attenuated with the adiabatic partition, which was more pronounced when the position of the partition was higher. Shi and Khodadadi (2013) studied numerically the same problem reported by Nag et al. (1993) with almost perfectly conducting partition on the hot wall, but with more extensive parametric details. Their dimensionless fin length was between 0.20 and 0.50 , which had seven positions along the hot wall and Rayleigh number was from $10^{4}$ to $10^{7}$. Since the fin was almost perfectly conducting and attached to the hot wall, the fins heating enhanced the convection while its blockage of the flow field suppressed it. The contributions of these two counter-acting mechanisms were not clearly quantified. Based on the numerical data, they proposed correlations to calculate Nusselt number as a function of relevant parameters for this particular case. Recently, Wu and Ching (2008, 2009) experimentally investigated the laminar natural convection in an air-filled partitioned square cavity with a temperature difference across the top and bottom walls. They found the flow pattern in the partitioned cavity was dependent on the top wall temperature of the cavity, the partition height and the partition location.

It should be noted that many of the studies in the open literature are rectangular and square cavity. The aim of the present study is to investigate numerically the buoyancy-induced flow and heat transfer mechanisms in a water-filled quadrantal cavity when the vertical wall of the cavity having solid adiabatic fin is hot while the lower surface is cold. The remaining curved wall is kept adiabatic. The work on quadrantal cavity has been done by Aydin (2011), and Yesiloz (2011) so far without considering adiabatic fin. Hence this is the first natural convection study in quadrantal cavity with solid adiabatic fin in the hot wall. 


\section{Problem Description}

The quadrantal cavity is filled with water inside shown in Fig.1. The vertical wall is the hot-wall, the bottom wall is the cold-wall and the curved wall is adiabatic. The height and the distance of the fin from the cooled bottom surface is represented by the two nondimensional number $\mathrm{h}$ and $\mathrm{c}$. Considering the effect of gravity, the natural convection process is studied by studying the heat transfer analysis with varying position and size of fin.

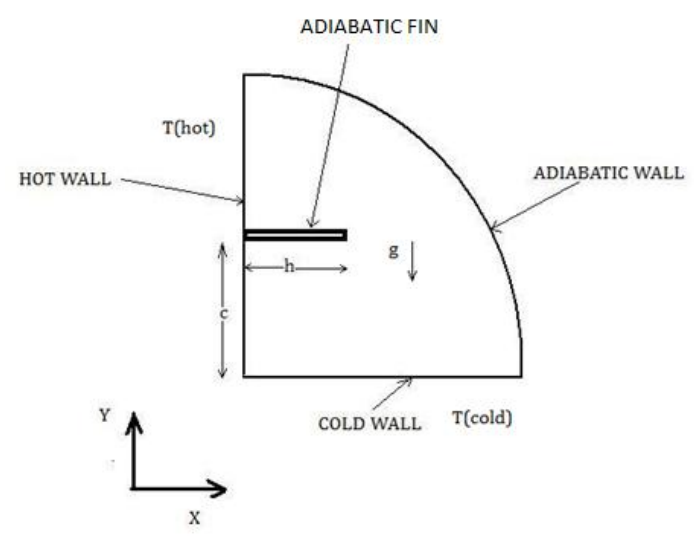

Fig. 1. Problem Description

\subsection{Governing Equations}

The continuity, momentum and energy equations for a two dimensional laminar flow of an incompressible Newtonian fluid is written. Following assumptions are made: there is no viscous dissipation, the cavity walls are impermeable, the gravity acts in negative $y$ direction, fluid properties are constant and fluid density variations are neglected except in the buoyancy term (the Boussinesq approximation) and radiation heat exchange is negligible because of very small temperature difference. Using non-dimensional variables defined in the nomenclature, the nondimensional governing equations are obtained as:

$\frac{\partial U}{\partial X}+\frac{\partial V}{\partial Y}=0$

$U \frac{\partial U}{\partial X}+\mathrm{V} \frac{\partial U}{\partial Y}=-\frac{\partial P}{\partial X}+\operatorname{Pr}\left(\frac{\partial^{2} U}{\partial X^{2}}+\frac{\partial^{2} U}{\partial Y^{2}}\right)$

$U \frac{\partial V}{\partial X}+\mathrm{V} \frac{\partial V}{\partial Y}=-\frac{\partial P}{\partial Y}+\operatorname{Pr}\left(\frac{\partial^{2} V}{\partial X^{2}}+\frac{\partial^{2} V}{\partial Y^{2}}\right)+\operatorname{RaPr} \theta$

$U \frac{\partial \theta}{\partial X}+\mathrm{V} \frac{\partial \theta}{\partial Y}=\frac{\partial^{2} \theta}{\partial X^{2}}+\frac{\partial^{2} \theta}{\partial Y^{2}}$

Appearing in Eq. (2) and Eq. (3), Pr and Ra are the Prandtl and Rayleigh numbers, respectively, which are defined as

$\operatorname{Pr}=\frac{\nu}{\alpha}, \operatorname{Ra}=\frac{g \beta H^{\mathbb{8}}\left(T_{h}-T_{\varepsilon}\right)}{v \alpha}$

where $\beta$ and $v$ are the thermal expansion coefficient and the kinematic viscosity of the fluid, respectively.

The non-dimensional parameters are listed as

$\mathrm{X}=\frac{x}{H}, \mathrm{Y}=\frac{y}{H}, \mathrm{U}=\frac{u}{\alpha / H}, \mathrm{~V}=\frac{v}{\alpha / H}$,

$$
\mathrm{P}=\frac{p \mathrm{H}^{\mathrm{z}}}{\rho \alpha^{\mathrm{z}}}, \theta=\frac{T-T_{c}}{T_{h^{2}}-T_{e}}
$$

\subsection{Boundary Conditions}

Through the introduction of the non-dimensional parameters into the physical boundary conditions illustrated in Fig. 1, the following non-dimensional boundary conditions are obtained:

On the adiabatic wall:

$\delta \theta / \delta n=0, \mathrm{U}=\mathrm{V}=0$ at $0<\mathrm{X}<1$ and $0<\mathrm{Y}<1$

On the bottom wall:

$\theta=0, \mathrm{U}=\mathrm{V}=0$ at $\mathrm{Y}=0$ and $0<\mathrm{X}<1$

On the vertical wall:

$\theta=1, \mathrm{U}=\mathrm{V}=0$ at $\mathrm{X}=0$ and $0<\mathrm{Y}<1$

\section{NUMERICAL APPROACH}

The continuity, momentum and the energy equations are solved using commercially available software FLUENT 6.3. Discretization of the momentum and energy equations is performed by a second order upwind scheme and pressure interpolation is provided by PRESTO scheme. Convergence criterion considered as residuals is admitted $10^{-5}$ for momentum and continuity equations and for the energy equation it is $10^{-12}$. In this study, the mesh is structured in such a way that the path of the heat lines, which intersect the isotherms spanning orthogonally from the isothermal hot bottom wall to the isothermal cold curved wall for the conduction solution, is considered.

The Nusselt number along the hot wall can be defined as

$\mathrm{Nu}_{\mathrm{r}}=\frac{q \times r}{T_{\text {hot }}-T_{\text {cold }}} \times \frac{\pi}{2} \times \frac{1}{k}$

Thus, we calculated Nusselt number manually using the Eq. (10).

\section{GRID INDEPENDENCY AND VALIDATION}

\subsection{Grid independency test}

In this study, four different mesh sizes $(40 \times 40,60 \times 60$, $80 \times 80$ and $100 \times 100)$ are adopted in order to check the mesh independence. A mesh structure without fin is shown in Fig.2. A detailed grid independence study has been performed, and results are obtained for the average Nusselt number, and the maximum values of the stream function, but any considerable changes were not obtained. Thus, a grid size of $80 \times 80$ is found to meet the requirements of both the grid independency study and the computational time limits which has shown in Table 1 . 
T. K. Bose et al. / JAFM, Vol. 6, No. 4, pp. 501-510, 2013.

Table 1 Grid independency test for enclosure without fin

\begin{tabular}{|c|c|c|c|c|}
\hline \multicolumn{6}{|c|}{$\left(\mathrm{T}_{\mathrm{h}}=279.2 \mathrm{k}, \mathrm{T}_{\mathrm{c}}=278.8 \mathrm{k}, \mathrm{Ra}=10^{4}\right)$} \\
\hline Mesh size & $40 \times 40$ & $60 \times 60$ & $80 \times 80$ & $100 \times 100$ \\
\hline$\Psi_{\mathrm{MAX}}$ & 2.01 & 2.0106 & 2.0263 & 2.0296 \\
\hline $\begin{array}{c}\text { Relative } \\
\text { error }(\%)\end{array}$ & \multicolumn{5}{|c|}{0.0298} & 0.7748 & 0.33 \\
\hline Nu (avg) & 5.9812 & 6.5737 & 7.1706 & 7.607 \\
\hline $\begin{array}{c}\text { Relative } \\
\text { error }(\%\end{array}$ & 9.013 & 8.3243 & 5.737 \\
\hline
\end{tabular}

The result obtained has been validated against the existing results for a inclined quadrantal cavity filled with water medium at $\phi=90^{\circ}$ which has been studied

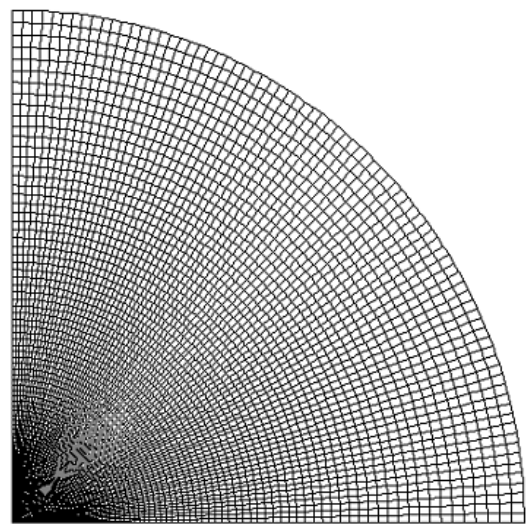

Fig. 2. Mesh Structure without fin

experimentally and numerically by Aydin (2011), and Yesiloz (2011). Figure 3 and Fig. 4 indicate that the result show good agreement with the literature.

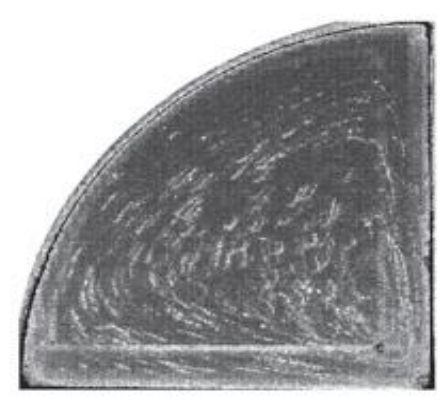

(a)

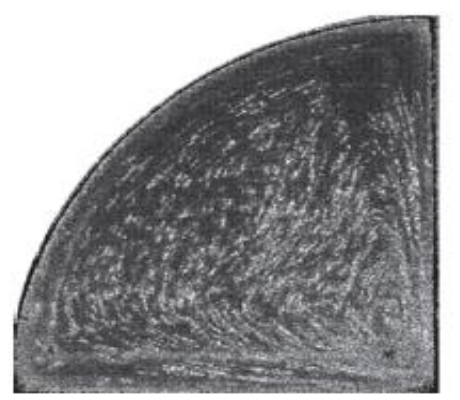

(b)

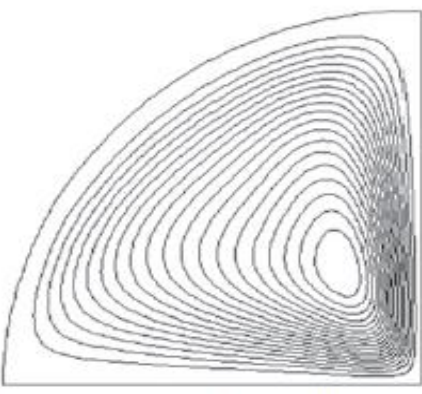

$\Psi_{\max }=4.20$

$\Psi_{\min }=0$

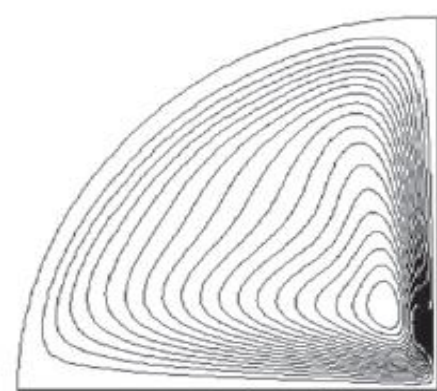

$\Psi_{\max }=5.89$

$\Psi_{\min }=0$

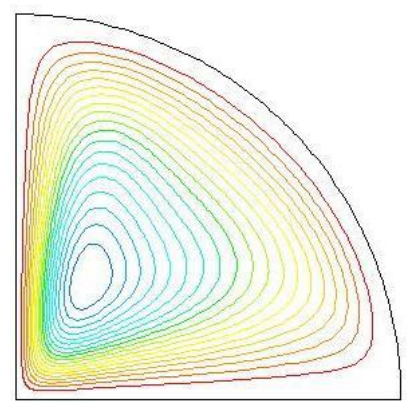

(a) $\quad \Psi=4.199$

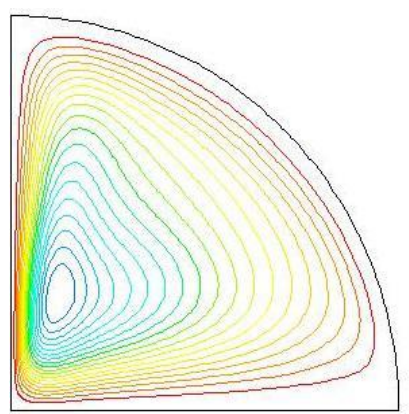

(b)

$\Psi=5.95$

Fig. 3. Experimental (extreme left), numerical (middle) streamlines from Aydin and Yesiloz and Numerical streamlines (extreme right) of present study at $\phi=90^{\circ}$ (a) $\mathrm{Ra}=1.7 \times 10^{5}$, (b) $\mathrm{Ra}=10^{6}$ 


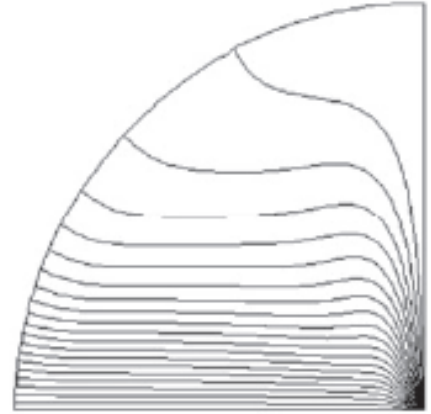

$\Delta \mathrm{T}=0.4 \mathrm{~K}$

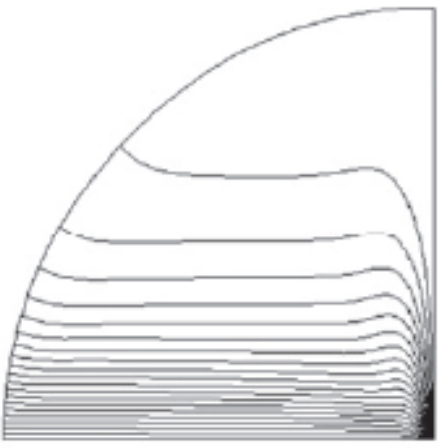

$\Delta \mathrm{T}=2.4 \mathrm{~K}$

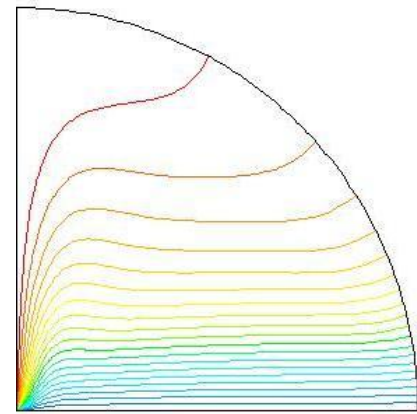

$\Delta \mathrm{T}=0.4 \mathrm{~K}$

(a)

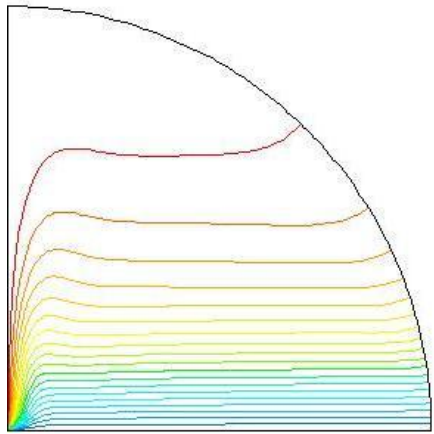

$\Delta \mathrm{T}=2.4 \mathrm{~K}$

(b)

Fig. 4. Numerical isotherms (left) from Aydin and Yesiloz and Numerical isotherms (right) of present study at $\phi=$

$$
90^{0} \text { (a) } \mathrm{Ra}=1.7 \times 10^{5} \text {, (b) } \mathrm{Ra}=10^{6}
$$

\section{RESULTS AND DISCUSSION}

Numerical analysis of laminar natural convection heat transfer and fluid flow is performed to obtain effects of Rayleigh number in an enclosure which is hot from the vertical wall. The curved wall is adiabatic in nature and the bottom wall has temperature less than that of the vertical wall. Results of flow fields and temperature distribution for different Rayleigh numbers, $R a$, dimensionless locations of thin fin $c$, dimensionless lengths of thin fin $h$, were plotted in this part of the study. Dimensionless height of the thin fin varied between 0.1 and 0.4. Also, dimensionless location of the thin fin on $y$-direction varied from 0.2 to 0.6. The range of the Rayleigh number, $R a$, was taken as $10^{4}$ to $10^{7}$.

Figures 5 and 6 shows the streamlines and isotherms for different Rayleigh numbers at $\mathrm{c}=0.4$ and $\mathrm{h}=0.2$ to 0.6 . As the length of the fin increases to $h=0.3$ or more, two cells are formed on the top and bottom of the fin in clockwise direction. Due to long fin the flow caused by buoyancy effect is obstructed and the flow bends around the fin as it moves upwards along the hot vertical wall and also forming two separate vortex. Thus the adiabatic fin divides the enclosure into two parts. The streamlines get crowded up near the bottom part of the vertical wall giving a boundary layer formation. The static temperature diagram shows concentration of isotherms near the cold bottom wall, suggesting that heat transfer rate is high near the vicinity of the bottom wall. Less packed isotherms are formed in the upper part of the partition that indicates lower heat transfer. At the lowest Rayleigh number $(R a$ $=10^{4}$ ), a relatively weak convective flow exists in the quadrantal cavity. As the Rayleigh number increases, the flow below the fin moves to the opening between the fin and curved wall, and a jet-like flow distribution is observed in this area. Isotherms show almost the same pattern for all Rayleigh numbers. Effects of the thin fin on isotherms become stronger as the Rayleigh number increases. For lower Rayleigh numbers, the convection intensity in the enclosure is very weak as evident from the values of the stream functions. It means that the viscous forces are more dominant than the buoyancy forces at lower $R a$ numbers. On the other hand, the fin length has a significant effect especially on the lower cell, which is located at the below of the fin. It enhances the strength of this cell due to the increasing strength of the jet flow.

Different positions of the thin fin are given in Fig. $7 \&$ 8 for $R a=10^{4}$ to $10^{7}, \mathrm{~h}=0.2$, no fin and $c=0.2,0.4$, 0.6. The first row of the Fig $5 \& 6$ shows streamlines and isotherms obtained numerically for no fin condition. At the lowest Rayleigh number $\mathrm{Ra}=10^{4}$, streamlines form a nearly centrally located single cell forming an elliptical shape, and corresponding isotherms exhibit the characteristics of quasiconduction. The hot fluid layer heated around the hot 
wall ascends upward by reason of decreasing density and the weak circulation in the enclosure. On the other hand, nearby the curve wall, the fluid layer with increased density moving downward sweeps the hot fluid layer forward. These mutual effects of the hot and cold fluid layers cause isotherms to widen. Further increases in $\mathrm{Ra}$, recirculation intensity increases to a degree that boundary layer formations are observed adjacent to the heated and cooled walls and heated flow impinges to the bottom wall. For the lower position of the fin as $c=0.2$, an elliptical shape single cell has formed at the lowest Rayleigh number $\mathrm{Ra}=10^{4}$. As increases Rayleigh number two cells are formed and also boundary layer formation occurs near the vertical wall and the cavity is divided into two parts. The higher rate of heat transfer occurs below the fin. For a further fin position, $c=0.4$, an elliptical-shaped cell is formed at the middle of the quadrantal enclosure at Rayleigh number $\mathrm{Ra}=10^{4}$, but a further increase of Rayleigh numbers formed two cells in the enclosure. The single cell were formed with $c=0.6$ near the horizontal wall. The isotherm plots are concentrated towards the bottom wall.

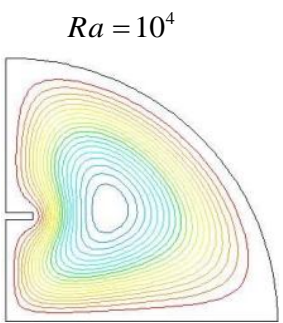

$\Psi=1.644$

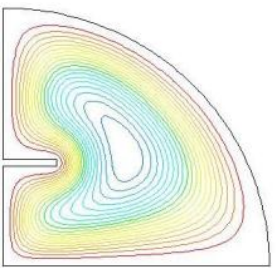

$\Psi=1.149$

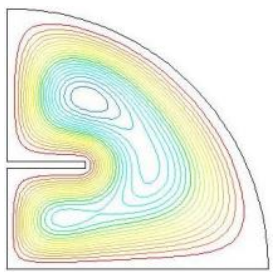

$\Psi=0.914$

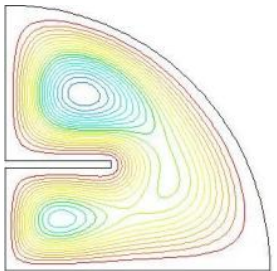

$\Psi=0.889$

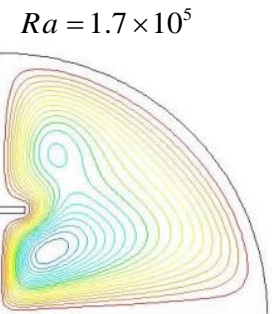

$\Psi=4.182$

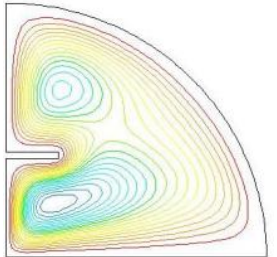

$\Psi=3.871$

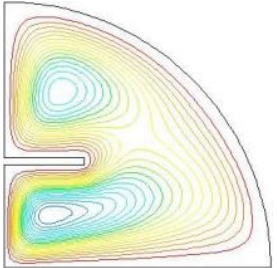

$\Psi=3.752$

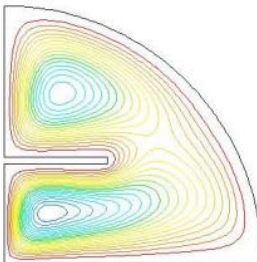

$\Psi=3.68$
$R a=10^{6}$

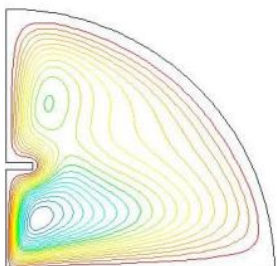

$\Psi=6.459$

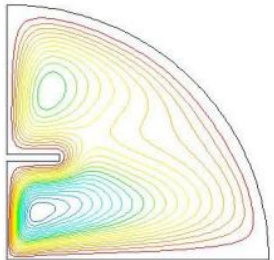

$\Psi=6.443$

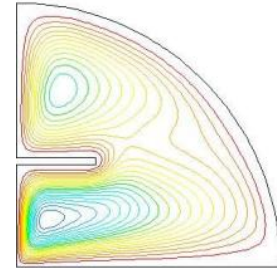

$\Psi=6.378$

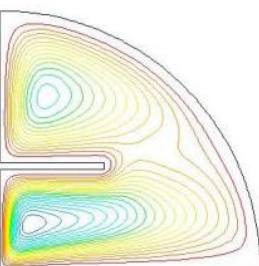

$\Psi=6.311$

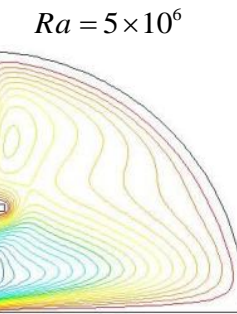

$\Psi=8.479$

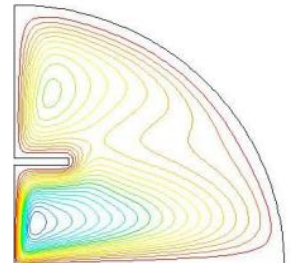

$\Psi=8.607$

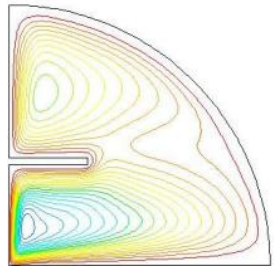

$\Psi=8.645$

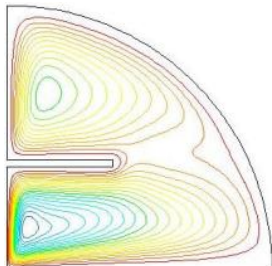

$\Psi=8.669$
$R a=10^{7}$

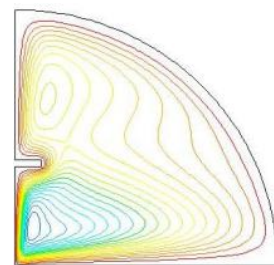

$\Psi=9.460$

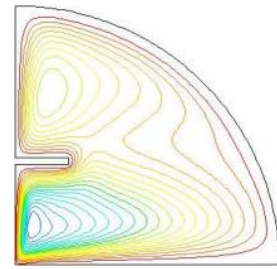

$\Psi=9.623$

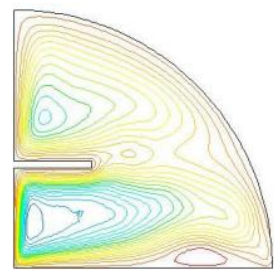

$\Psi=9.673$

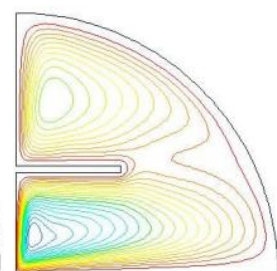

$\Psi=9.721$

Fig. 5. Streamlines for dimensionless fin location, $\mathrm{c}=0.4$, column wise different Raleigh Numbers, $R a=10^{4}$, $R a=1.7 \times 10^{5}, R a=10^{6}, R a=5 \times 10^{6}, R a=10^{7}$; row wise different dimensionless fin length, $\mathrm{h}=0.1, \mathrm{~h}=0.2$, $\mathrm{h}=0.3, \mathrm{~h}=0.4$ 
T. K. Bose et al. / JAFM, Vol. 6, No. 4, pp. 501-510, 2013.
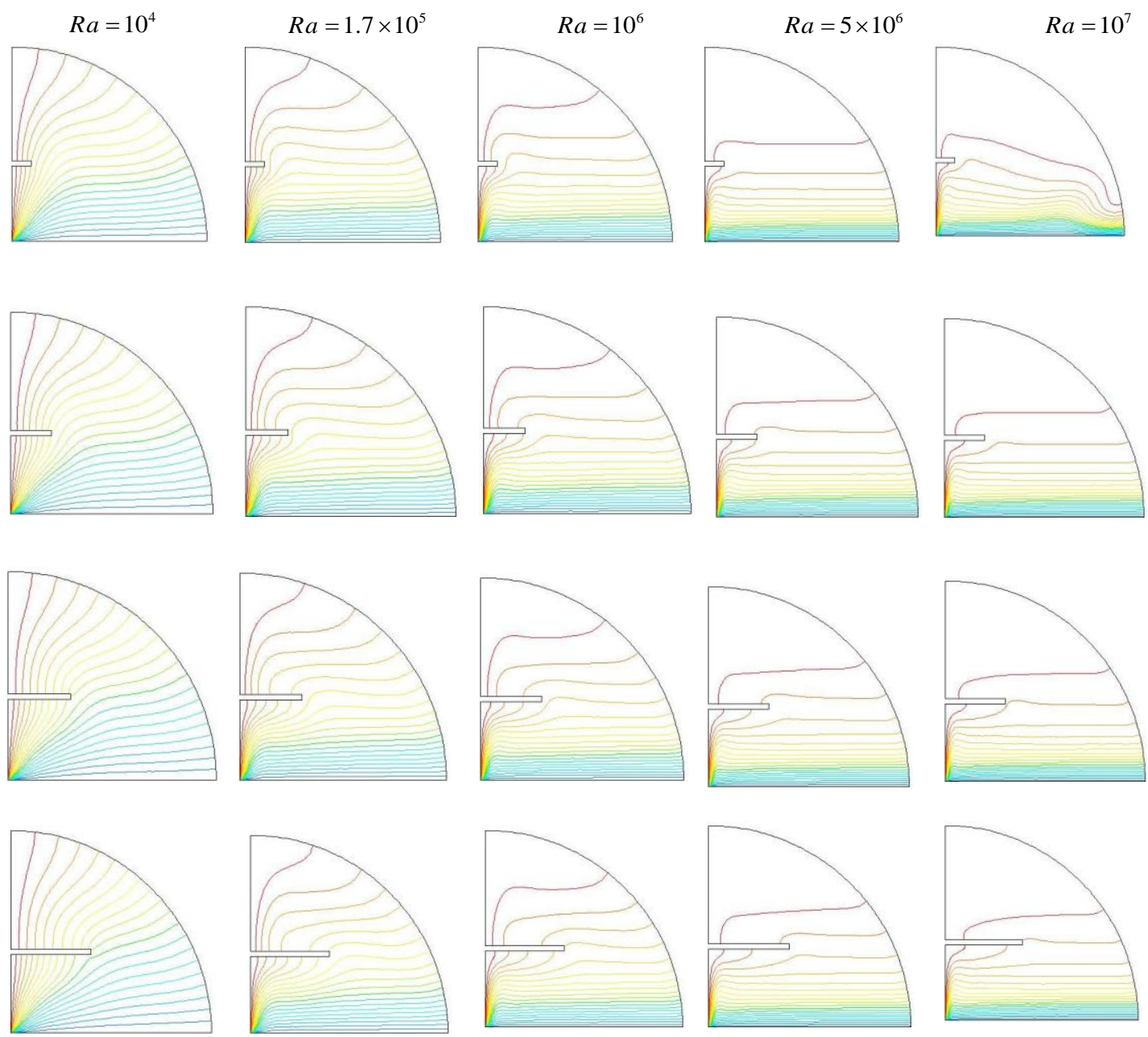

Fig. 6. Isotherms for dimensionless fin location, $\mathrm{c}=0.4$, column wise different Raleigh Numbers, $R a=10^{4}$, $R a=1.7 \times 10^{5}, R a=10^{6}, R a=5 \times 10^{6}, R a=10^{7}$; row wise different dimensionless fin length, $\mathrm{h}=0.1, \mathrm{~h}=0.2, \mathrm{~h}=0.3$, $\mathrm{h}=0.4$
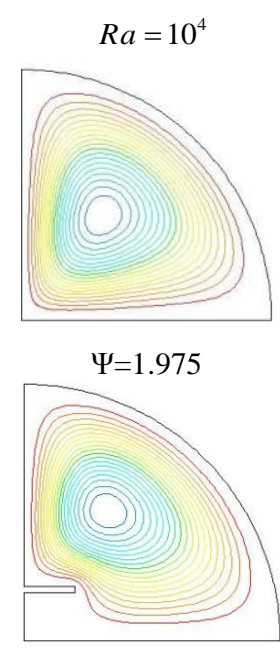

$\Psi=1.647$

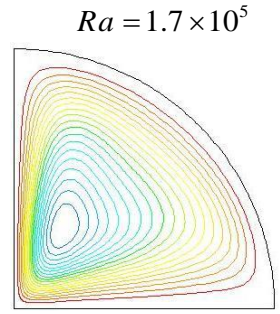

$\Psi=4.199$

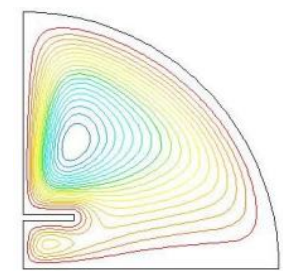

$\Psi=3.810$

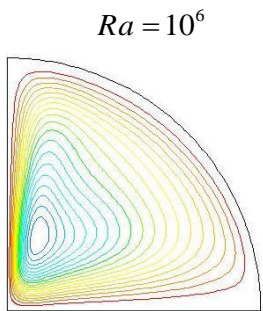

$\Psi=5.951$

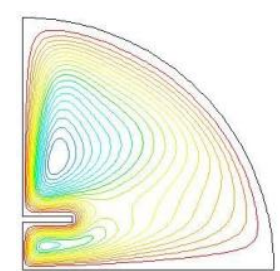

$\Psi=5.229$

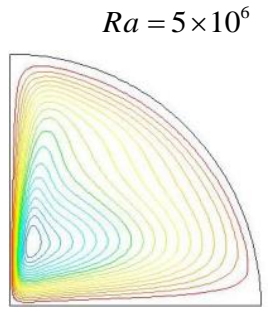

$\Psi=7.296$

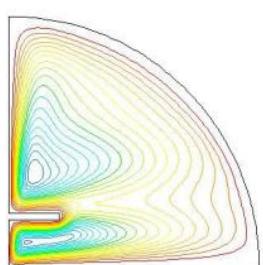

$\Psi=7.120$

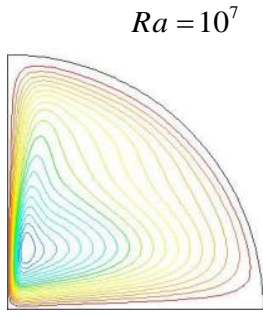

$\Psi=8.072$

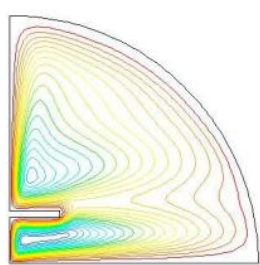

$\Psi=8.665$ 


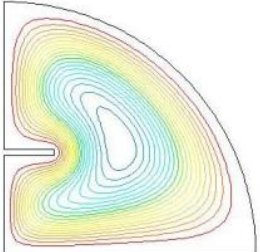

$\Psi=1.149$

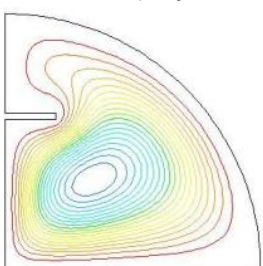

$\Psi=\Psi=1.891$

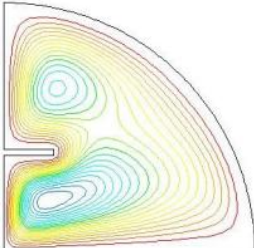

$\Psi=3.891$

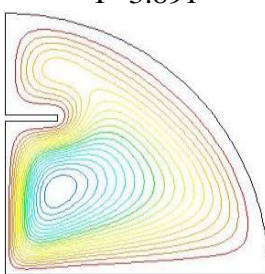

$\Psi=4.122$

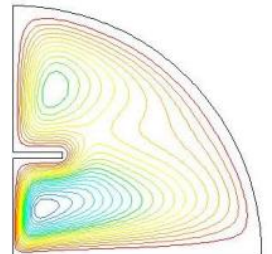

$\Psi=6.438$

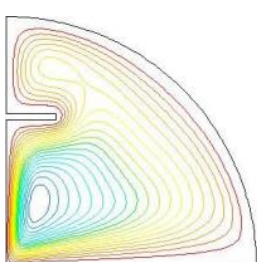

$\Psi=6.557$

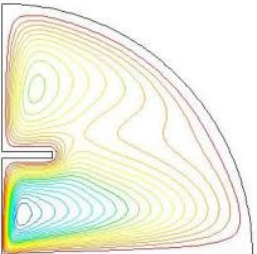

$\Psi=8.61$

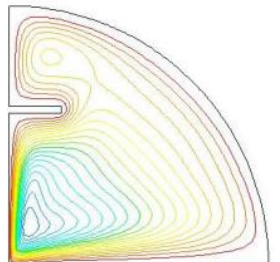

$\Psi=8.247$

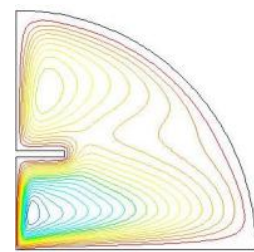

$\Psi=9.626$

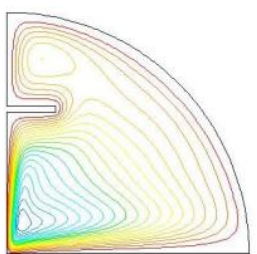

$\Psi=9.342$

Fig. 7. Streamlines for dimensionless fin height, $\mathrm{h}=0.2$, column wise different Raleigh Numbers, $R a=10^{4}$, $R a=1.7 \times 10^{5}, R a=10^{6}, R a=5 \times 10^{6}, R a=10^{7}$; row wise different dimensionless fin location, no fin, $\mathrm{c}=0.2$, $\mathrm{c}=0.4, \mathrm{c}=0.6$
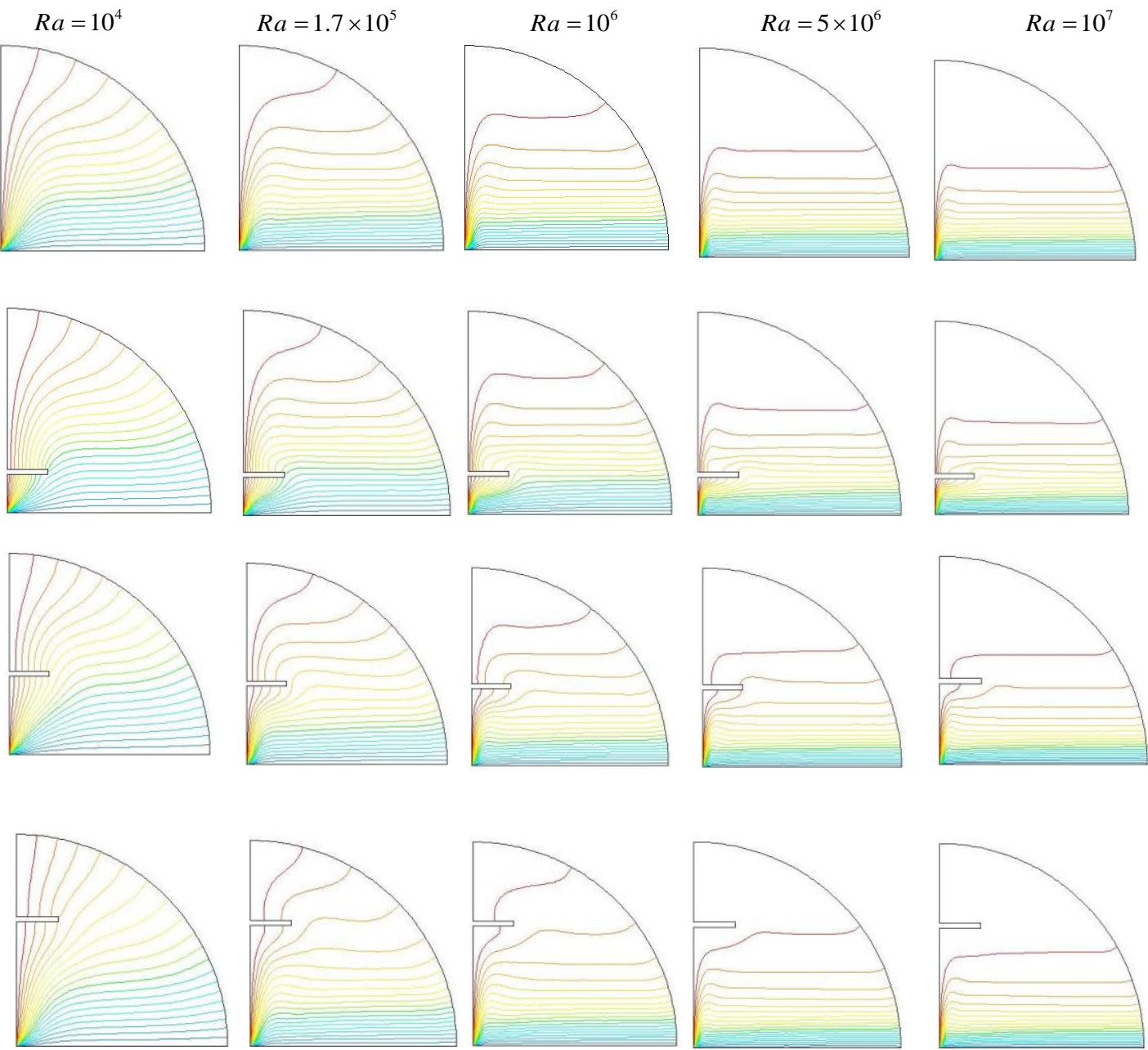

Fig. 8. Streamlines for dimensionless fin height, $\mathrm{h}=0.2$, column wise different Raleigh Numbers, $R a=10^{4}$, $R a=1.7 \times 10^{5}, R a=10^{6}, R a=5 \times 10^{6}, R a=10^{7}$ 
T. K. Bose et al. / JAFM, Vol. 6, No. 4, pp. 501-510, 2013.

Fig 9(a) shows the effects of dimensionless fin location on mean Nusselt number. For small Rayleigh numbers, $\mathrm{Nu}$ number values are almost equal to each other due to quasi-conductive regime. However, as expected, these values increase as $R a$ number increases. Dimensionless location of the fin plays an important role for higher Rayleigh numbers.

The value of the mean Nusselt number as a function of the dimensionless fin height is presented in Fig. 9(b). for $c=0.4$. The variations of the mean Nusselt number are shown for different Rayleigh numbers. As the dimensionless fin height increases, the value of the mean Nusselt number almost remains constant. When these values are compared to the no-fin condition, it is observed that the no-fin condition gives more heat transfer.

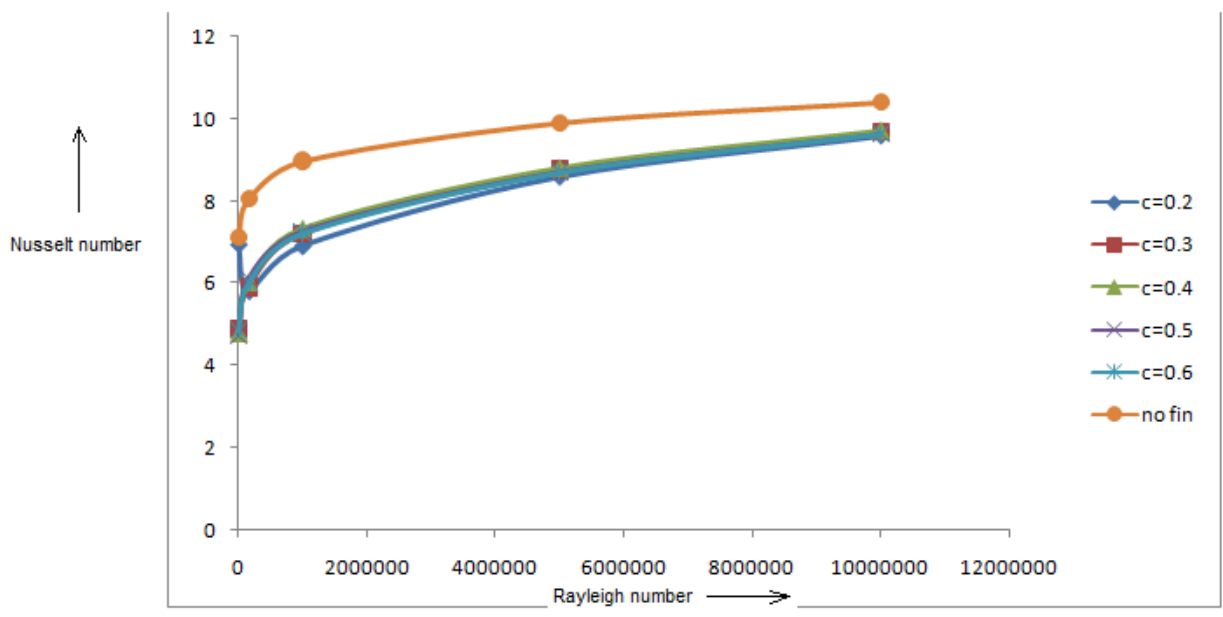

Fig. 9(a). Variation of mean Nu no. with Rayleigh number $\left(10^{4}\right.$ to $\left.10^{7}\right)$ for different fin locations $(\mathrm{c}=0.2,0.3,0.4,0.5$, 0.6 and no fin) for $\mathrm{h}=0.2$

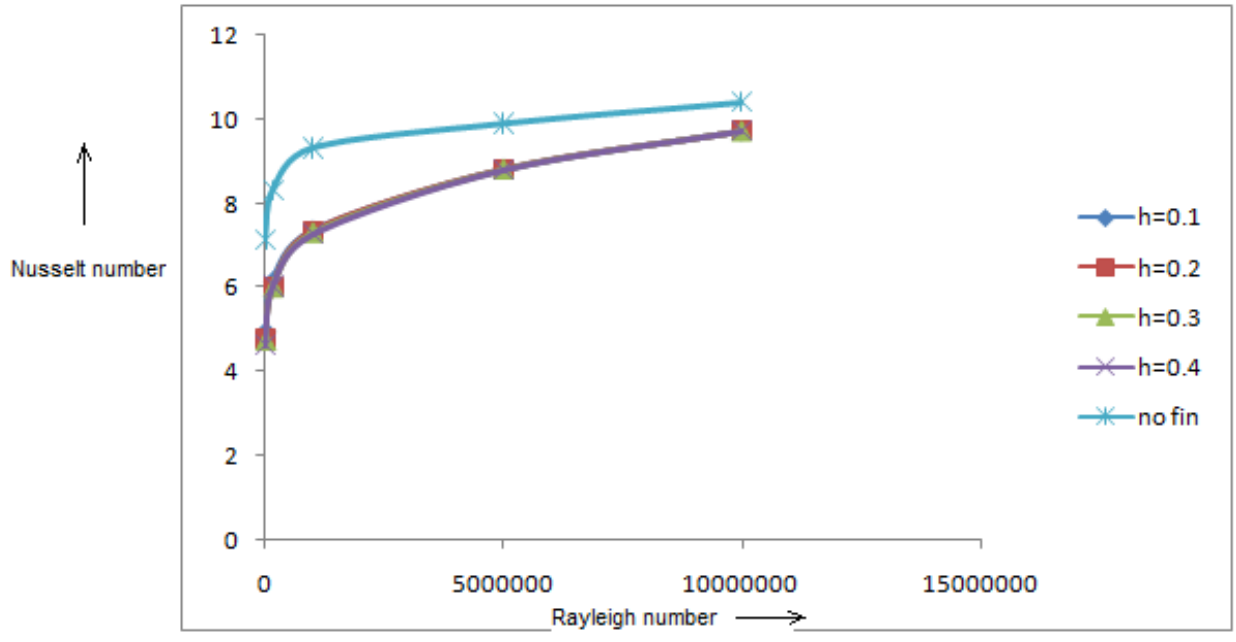

Fig. 9(b). Variation of mean Nu no. with Rayleigh number $\left(10^{4}\right.$ to $\left.10^{7}\right)$ for different fin locations $(\mathrm{h}=0.1,0.2,0.3,0.4$ and no fin) for $\mathrm{c}=0.4$

\section{Conclusions}

Numerical study has been performed to analyze the flow and temperature fields as well as the heat transfer rate on laminar natural convection in quadrentral cavity filled with water having finned hot vertical wall and cold bottom wall. The effects of Rayleigh number $\left(10^{4}\right.$ to $10^{7}$ ), non-dimensional fin location ( $c=0.2$ to 0.6 ) and non-dimensional fin height $(\mathrm{h}=0.1$ to 0.4$)$ have been studied.

The result of the numerical analysis lead to the following conclusions:

1) Heat transfer increases with increase of Rayleigh number. Values of stream function (flow strength) also increase with the increasing of Rayleigh number. 
2) Non-dimensional fin location changes the shape of vortices and enhances the strength of the flow.

3) With the increasing of dimensionless fin length, the fin causes blockage effect for the flow.

4) It was observed that the fin can be a passive control parameter for heat transfer and fluid flow.

\section{REFERENCES}

Ampofo, F. (2004). Turbulent natural convection in an air filled partitioned square cavity, Int. J. Heat Fluid Flow, 25, 103-114.

Ampofo, F. (2005) Turbulent natural convection of air in a non-partitioned or partitioned cavity with differentially heated vertical and conducting horizontal walls, Exp. Thermal Fluid Sci., 29, 137157.

Aydin, O. and G. Yesiloz (2011). Natural Convection in a Quadrantal Cavity Heated and Cooled on Adjacent Walls, J. Heat Transfer, 133, 052501-7.

Aydin, O., and G. Yesiloz (2011). Natural Convection in an inclined Quadrantal Cavity Heated and Cooled on Adjacent Walls, Exp. Therm. Fluid Sci., $35,1169-1176$.

Bajorek, S.M., J.R. Lloyd (1982). Experimental investigation of natural convection in partitioned enclosures, J. Heat Transfer, 104, 527-532.

Bilgen, E. (2002). Natural convection in enclosures with partial partitions, Renewable Energy, 26, 257270.

Frederick, R.L. (1989). Natural convection in an inclined square cavity with a partition attached to its cold wall, Int. J. Heat Mass Transfer, 32, 87-94.

Frederick, R.L. (1989). A. Valencia, Heat transfer in a square cavity with a conducting partition on its hot wall, Int. Commun. Heat Mass Transfer, 16, 347354.

Frederick, R.L. (1989). A. Valencia, Heat transfer in a square cavity with a conducting partition on its hot wall, Int. Commun. Heat Mass Transfer, 16, 347354.

Ganzarolli, M. M. and L. F. Milanez (1995). Natural Convection in Rectangular Enclosures Heated From Below and Symmetrically Cooled from Sides, Int. J. Heat Mass Transfer, 38, 1063-1073.

Jetli, R., S. Acharya and E. Zimmerman (1986). Influence of baffle location on natural convection in a partially divide enclosure, Numer. Heat Transfer, $10,521-536$.

Lin, N.N. and A. Bejan (1983). Natural convection in a partially divided enclosure, Int. J. Heat Mass Transfer. 26, 1867-1878.
Nag, A., A. Sarkar and V.M.K. Sastri (1993). Natural convection in a differentially heated square cavity with a horizontal partition plate on the hot wall, Comput. Meth Appl. Mech. Eng., 110, 143-156.

Nishimura, T., M. Shiraishi and Y. Kawamura (1987). Natural convection heat transfer in enclosures with an off-center partition, Int. J. Heat Mass Transfer, $30,1756-1758$.

November, M. and M. W. Nansteel (1987). Natural Convection in Rectangular Enclosures Heated From Below and Cooled Along One Side, Int. J. Heat Mass Transfer, 30, 2433-2440.

Nowak, E.S. and M.H. Novak (1994). Vertical partitions in slender rectangular cavities, Int. J. Heat Fluid Flow, 15, 104-110.

Oosthuizen, P.L. and J.T. Paul (1985). Free convection heat transfer in a cavity fitted with a horizontal plate on the cold wall, in: S.M. Shenkman et al. (Eds.), Advances in Enhanced Heat Transfer, 43, 101-107.

Ravi, M.R., R.A.W.M. Henkes and C.J. Hoogendoorn (1994). On the high-Rayleigh-number structure of steady laminar natural-convection flow in a square enclosure, J. Fluid Mech., 262, 325-351.

Shi, X. and J.M. Khodadadi (2003). Laminar natural convection heat transfer in a differentially heated square cavity due to a thin fin on the hot wall, $J$. Heat Transfer, 125, 624-634.

Turkoglu, H. and N. Yucel (1996). Natural convection heat transfer in enclosures with conducting multiple partitions and side walls, Heat Mass Transfer. 32, $1-8$.

Wu, W., D. Ewing and C.Y. Ching (2006). The effect of the top and bottom wall temperatures on the laminar natural convection in an air-filled square cavity, Int. J. Heat Mass Transfer, 49, 1999-2008.

Wu, W., D. Ewing, C.Y. Ching (2008). Investigation of a large top wall temperature on the natural convection plume along a heated vertical wall in a square cavity, Int. J. Heat Mass Transfer, 51, 15511561.

Wu, W. and C.Y. Ching (2009). The effect of the top wall temperature on the laminar natural convection in rectangular cavities with different aspect ratios, J. Heat Transfer, 131, 1-11.

Wu. W., C.Y. Ching (2008). The effect of partitions on the laminar natural convection in a square cavity, ASME Summer Heat Transfer Conference, Jacksonville, Florida, USA.

Yucel, N. and H. Turkoglu (1998). Numerical analysis of laminar natural convection in enclosures with fins attached to an active wall, Heat Mass Transfer, 33, 307-314. 